Casos Clínicos

Arch. Esp. Urol. 2009; 62 (9): 758-760

\section{ASOCIACIÓN DE CISTOPATIA EOSINOFILICA CON CARCINOMA TRANSICIONAL VESICAL INFILTRANTE}

\author{
Nicolás Alberto Cruz Guerra, María Montserrat \\ Chimeno Viñas' Inmaculada Ursúa Sarmiento², Emilia \\ Martínez Velado' y Alberto del Valle Manteca?2.
}

Servicio de Urología. Servicio de Medicina Interna' y Servicio de Anatomía Patológica². Complejo Hospitalario de Zamora. Zamora. España.

Resumen.- OBJETIVOS: Presentación de un caso de cistopatía eosinofílica asociada a carcinoma vesical infiltrante de células transicionales en adulto.

MÉTODO: Describimos el caso de una paciente mujer de 79 años que refiere dolor meso e hipogástrico de varios meses de evolución, y a la que se detecta en escáner abdominal un engrosamiento parietal vesical durante el estudio de descarte de patología digestiva por parte del Servicio de Medicina Interna. La cistoscopia permitió confirmar dicho hallazgo, junto a zonas de mayor protrusión endoluminal. La biopsia fría mostró la existencia de infiltrado inflamatorio con componente eosinofílico. Se indicó resección transuretral.
RESULTADOS: El estudio anatomopatológico resultó compatible con carcinoma transicional vesical infiltrante de alto grado. Desarrollo de metástasis pulmonares múltiples y éxitus de la paciente cinco meses después de la cirugía.

CONCLUSIONES: Infrecuencia de las descripciones de aparición coincidente de ambas entidades referidas. Destacamos la importancia de una correcta filiación de lesiones endoluminales aparentemente "pseudotumorales".

Palabras clave: Cistopatía eosinofílica. Carcinoma de células transicionales. Cirugía.

Summary.- OBJECTIVES: To report a case of eosinophilc cystopathy associated to infiltrative transitional-cell carcino ma of the bladder in an adult.

METHODS: We describe the case of a 79-year-old wo man who complains of meso- and hipogastric pain since several months before, and to whom a parietal thickening of the bladder was detected by means of abdominal CT scan during the diagnostic work up for a digestive disease indicated by the Internal Medicine Service. Cistoscopy confirmed that finding, together with areas of greater endolu minal protrusion. Cold biopsy showed an inflammatory in filtrate with eosinophilic component. Transurethral resection was indicated.

RESUITS: The pathological assessment was compatible with high-grade infiltrative transitional carcinoma of the bladder. The patient developed multiple lung metastases and died five months after surgery.

CONCLUSIONS: Reports describing the coincidence of both entities are infrequent. We emphasize the importance of a correct filiation of apparently "pseudotumoral" endoluminal lesions.

Keywords: Eosinophilic cystopathy. Transitional-cell carcinoma. Surgery.

\section{INTRODUCCIÓN}

\section{CORRESPONDENCIA}

Nicolás Alberto Cruz Guerra

Av. de los Reyes Católicos, 5 bajo izda. 49021 . Zamora. (España).

ncruz_g@hotmail.com

Trabajo recibido: 15 de abril 2009. Presentamos un caso de este tipo, con la particularidad
La cistopatía eosinofilica es una entidad con referencias cuantitativamente limitadas en la literatura urológica. añadida de su asociación a carcinoma transicional infiltrante vesical.

\section{CASO CLÍNICO}

Paciente mujer de 79 años con insuficiencia renal crónica terminal secundaria a nefropatía diabética, en programa de hemodiálisis desde hacía cuatro años, y antecedentes de alergia a penicilina, que refiere molestias abdominales inespecíficas de varios meses de evo- 
lución, de predominio en meso e hipogastrio, de intensidad leve-moderada, a la que se le detecta, durante el estudio de dichas manifestaciones por parte del Servicio de Medicina Interna, una imagen de engrosamiento parietal vesical difuso en escáner abdominopélvico (Figura 1).

La exploración física sólo permitió constatar molestias coincidentes con la palpación bimanual abdominovaginal, así como cierta disminución de la movilidad vesical. Los parámetros analíticos básicos resultaron dentro de la normalidad, salvo una glucemia de $146 \mathrm{mg} / \mathrm{dL}$, creatinina sérica de $9,5 \mathrm{mg} / \mathrm{dL}$ y discretas leucocitosis y neutrofilia, 11.600 leucos/ $\mathrm{mL}$ con $74 \%$ de neutrófilos. Se indicó la realización de cistoscopia, confirmándose la existencia de un engrosamiento difuso parietal, que afectaba especialmente a la base y ambas caras laterales vesicales, con algunas áreas de mayor protrusión. Se tomó biopsia fría, cuyo estudio permitió objetivar un infiltrado inflamatorio significativo, con componente eosinofílico (Figura 2).

Ante los hallazgos mencionados, y dada la persistencia de la clínica de la paciente, se optó por resección transuretral de las áreas afectas. El estudio anatomopatológico demostró la existencia de un carcinoma transicional de alto grado que infiltraba en profundidad la pared vesical (Figura 3). Dados los condicionantes de la paciente, así como un estado basal no satisfactorio, se acordó con su familia no someterla a cirugías exeréticas agresivas, siéndole administrada radioterapia pélvica.

A los dos meses de la cirugía se detectó la presencia de metástasis pulmonares múltiples bilaterales en TAC toracoabdominopélvico de control. El deterioro posterior de la paciente fue progresivo, produciéndose el éxitus tres meses después de dicha prueba.

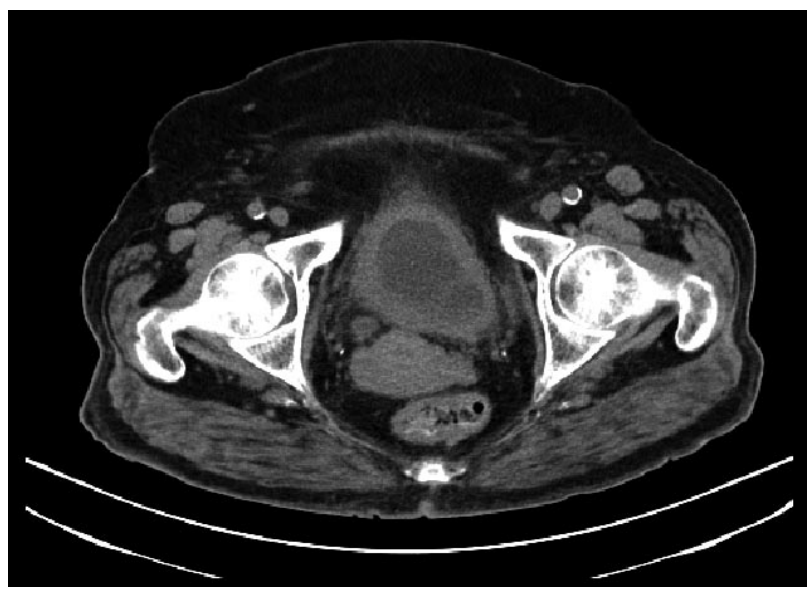

FIGURA 1. TAC abdominopélvica en la que se aprecia engrosamiento parietal vesical difuso, más evidente en pared lateral derecha.

\section{DISCUSIÓN}

La cistopatía eosinofílica constituye una entidad poco frecuente, de tal forma que hasta el inicio de la década actual la casuística publicada sólo constaba de algo más de un centenar de casos (1). Su escasa incidencia dificulta la caracterización de su perfil epidemiológico, si bien el rango etario de los casos recogidos en la literatura es notablemente amplio, abarcando desde el periodo neonatal hasta la novena década de la vida. No parece existir mayor predisposición de sexo, excepto en edad pediátrica, durante la cual se presenta con mayor frecuencia en varones (1).

La etiología de la cistopatía eosinofílica resulta aún desconocida. Se han emitido hipótesis que toman como base las reacciones de hipersensibilidad inmediata, o como respuesta ante determinados complejos antigénicos bacterianos. En este sentido se ha querido establecer diferenciación entre aquellos casos, especialmente mujeres, con afectación vesical difusa en los que se verifica un componente atópico, asma crónica, polinosis, frente a un grupo de pacientes en los que la cistopatía mencionada es vinculada a una variedad de posibilidades etiológicas: fármacos, instilaciones endovesicales de agentes citostáticos o inmunoterápicos, parasitosis, cuerpos extraños, radiaciones y resecciones transuretrales, entre otros (2). Los déficits estrogénicos asociados a historia de alergia han sido postulados como factores adyuvantes en pacientes con anorexia nerviosa (3). La coexistencia de cistopatía eosinofílica con carcinoma transicional vesical ha sido descrita, aunque las citas bibliográficas son escasas (4).

Las manifestaciones clínicas principales son las propias de una sintomatología miccional irritativa, con predominio de polaquiuria, disuria y dolor suprapúbico (1). La

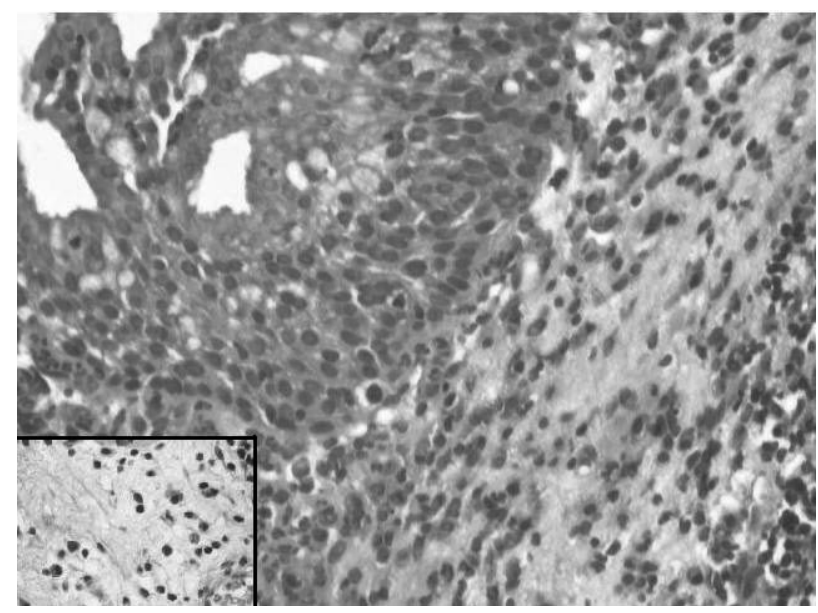

FIGURA 2. Infiltrado inflamatorio parietal vesical con componente eosinófilo $(H$ y $E, \times 20)$ y

detalle de este último, en área edematosa $(H$ y $E, x$ 40) (recuadro inferior izquierdo). 
hematuria macro o microscópica puede estar presente, sobre todo en aquellos casos de asociación neoplásica, aunque curiosamente en el caso que describimos la intensidad de los signos y síntomas resultó ser significativamente limitada. La eosinofilia, asociada o no a eosinofiluria, se verifica en un $25-50 \%$ de pacientes adultos, sobre todo en aquellos con componente alérgico. Su aparición resulta aun más frecuente en la infancia, si bien en esta etapa su asociación con alergias es rara (2).

Las técnicas de imagen, principalmente ecografía y tomografía axial computerizada, no aportan especificidad de cara al diagnóstico de la cistopatía eosinofílica (5), pero sí mantienen su valor para la detección de formas pseudotumorales, siendo por tanto indicativas de exploraciones complementarias ante la posibilidad de albergar una verdadera neoplasia. La cistoscopia, en este sentido, permite identificar placas amarillentas con posible ulceración superficial (6), con carácter focal o difuso (este último como forma más frecuente de afectación en el sexo femenino), aunque las morfologías irregulares, con diferentes grados de protrusión también han sido descritas $(7,8)$, siendo estos los casos en los que cobra su máxima relevancia la correcta filiación histológica de las muestras biópsicas. Desde un punto de vista microscópico, la característica fundamental es el infiltrado inflamatorio localizado en lámina propia y muscular, con componente eosinófilo significativo (8), pudiendo adoptar patrones granulomatosos.

Varias han sido las opciones terapéuticas empleadas en la cistopatía eosinofílica, con carácter más o menos conservador: eliminación de potenciales antígenos, asociación de corticoides y antihistamínicos, con/sin antibióticos, o intervencionista, resecciones endoscópicas, pudiendo combinarse ambas opciones (1). El control evolutivo de los pacientes es claramente recomendable, dada su tendencia a recidivar (9), llegando incluso a

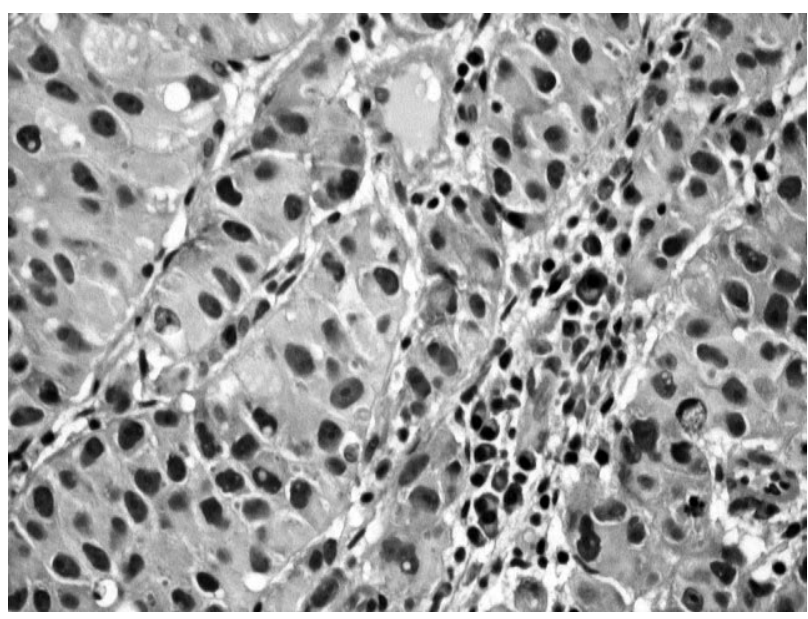

FIGURA 3. Células de carcinoma transicional vesical, pleomórficas, con patrón de cromatina nuclear y nucleolos prominentes $(H$ y $E, x 20)$. originar fibrosis vesical severa (10). En este último caso, así como en sujetos con hematurias recidivantes como manifestación predominante, tendrían su indicación actitudes más agresivas, cistectomía parcial o total, y también en aquellos pacientes que asocian neoplasia infiltrante, salvo algunos como la aquí presentada, en los que las únicas opciones aplicables son meramente paliativas, dada la rápida evolución del tumor y consecuente pronóstico infausto.

\section{CONCLUSIÓN}

Si bien la aparición coincidente de cistopatía eosinofílica y carcinoma transicional vesical es infrecuente, constituye un ejemplo de la importancia de una correcta filiación de lesiones endoluminales aparentemente "pseudotumorales" que pudieran albergar un verdadero proceso neoplásico maligno.

\section{BIBLIOGRAFIA Y LECTURAS \\ RECOMENDADAS (*lectura de interés $y^{* *}$ lectura fundamental)}

**1. Van Den Ouden D. Diagnosis and management of eosinophilic cystitis: a pooled analysis of 135 cases. Eur Urol, 2000; 37: 386.

*2. Popert R J M, Ramsay J W A. Owen R A, et al. Eosinophilic cystitis mimicking invasive bladder tumour: discussion paper. J. R. Soc. Med, 1990; 83: 776.

3. Philip J, Ali F S, Zakhour H D, et al. Severe eosinophilic cystitis in a woman with anorexia nervosa. Int. J. Urol, 2006; 13: 1132.

*4. Castillo Jimeno J M, Gonzalez de Garibay A S, Ruiz Rubio J L, et al. Cistitis eosinofílica asociada con carcinoma de células transicionales: a propósito de un caso. Arch Esp Urol, 1992; 45: 151.

5. Wong You Cheong J J, Woodwar P J, Manning M A, et al. From the archives of the AFIP: Inflammatory and nonneoplastic bladder masses: radiologic-pathologic correlation. Radiographics, 2006; 26: 1847.

6. Blas Marin M, Rioja Sanz C. Cistitis intersticial y otras cistopatías. En: Jimenez Cruz J F, Rioja Sanz LA, editores. Tratado de Urología. $2^{\mathrm{a}}$ ed. Barcelona: Prous Science; 2006. Cap. 75, p. 1417-34.

7. Kiliç S, Erguvan R, Ipek D, et al. Eosinophilic cystitis. A rare inflammatory pathology mimicking bladder neoplasms. Urol Int, 2003; 71: 285.

8. Salman M, Al Ansari A A, Talib R A, et al. Eosinophilic cystitis simulating invasive bladder cancer: a rela diagnostic challenge. Int Urol Nephrol, 2006; 38: 545 .

*9. Teegavarapu P S, Sahai A, Chandra A, et al. Eosinophilic cystitis and its management. Int J Clin Pract, 2005; 59: 356.

10. Slama A, Khouni H, Sriha B, et al. Bladder fibrosis caused by eosinophilic cystitis. Ann Urol, 2003; 37 : 272. 